\title{
CARDINAL ARITHMETIC FOR SKEPTICS
}

\author{
SAHARON SHELAH
}

When modern set theory is applied to conventional mathematical problems, it has a disconcerting tendency to produce independence results rather than theorems in the usual sense. The resulting preoccupation with "consistency" rather than "truth" may be felt to give the subject an air of unreality. Even elementary questions about the basic arithmetical operations of exponentiation in the context of infinite cardinalities, like the value of $2^{\aleph_{0}}$, cannot be settled on the basis of the usual axioms of set theory (ZFC).

Although much can be said in favor of such independence results, rather than undertaking to challenge such prejudices, we have a more modest goal; we wish to point out an area of contemporary set theory in which theorems are abundant, although the conventional wisdom views the subject as dominated by independence results, namely, cardinal arithmetic.

To see the subject in this light it will be necessary to carry out a substantial shift in our point of view. To make a very rough analogy with another generalization of ordinary arithmetic, the natural response to the loss of unique factorization caused by moving from $\mathbb{Z}$ to other rings of algebraic integers is to compensate by changing the definitions, rescuing the theorems. Similarly, after shifting the emphasis in cardinal arithmetic from the usual notion of exponentiation to a somewhat more subtle variant, a substantial body of results is uncovered that leads to new theorems in cardinal arithmetic and has applications in other areas as well. The first shift is from cardinal exponentiation to the more general notion of an infinite product of infinite cardinals; the second shift is from cardinality to cofinality; and the final shift is from true cofinality to potential cofinality (pcf). The first shift is quite minor and will be explained in $\S 1$. The main shift in viewpoint will be presented in $\S 4$ after a review of basics in $\S 1$, a brief look at history in $\S 2$, and some personal history in $\S 3$. The main results on pcf are presented in $\S 5$. Applications to cardinal arithmetic are described in $\S 6$. The limitations on independence proofs are discussed in $\S 7$, and in $\S 8$ we discuss the status of two axioms that arise in the new setting. Applications to other areas are found in $\S 9$.

The following result is a typical application of the theory.

Theorem A. If $2^{\aleph_{n}}<\aleph_{\omega}$ for all $n$ then $2^{\aleph_{\omega}}<\aleph_{\omega_{4}}$.

The subscript 4 occurring here is admittedly very strange. Our thesis is that the theorem cannot really be understood in the framework of conventional cardinal arithmetic, but that it makes excellent sense as a theorem on pcf. Another way of putting the matter is that the theory of cardinal arithmetic involves two quite different aspects, one of which is totally independent of the usual axioms

Received by the editors October 1, 1990 and, in revised form, May 27, 1991.

1991 Mathematics Subject Classification. Primary 03E10; Secondary 03-03, 03E35.

Research partially supported by the BSF; Pub. no. 400A. 
of set theory, while the other is quite amenable to investigation on the basis of ZFC. Since the usual approach to cardinal arithmetic mixes these two aspects, the independence results mask the theorems and the occasional result that survives this interference then looks quite surprising.

Of course, the most severe skeptics will even deny the mathematical content of Cantor's theorem $\left(2^{\aleph_{0}}>\aleph_{0}\right)$. To these we have nothing to say at all, beyond a reasonable request that they refrain from using the countable additivity of Lebesgue measure.

Most of the results reported here were obtained in the past three years and are expected to appear in a projected volume to be published by Oxford University Press.

\section{BASICS}

The reader is assumed to be comfortable with the axiom system ZFC or an equivalent formulation of axiomatic set theory including the Axiom of Choice, though knowing naive set theory is enough for reading almost everything . In particular we have the notions of ordinal, cardinal, and cardinality $|A|$ of a set $A$, the identification of ordinals with sets of ordinals, of cardinals with "initial" ordinals, and hence also with sets of ordinals. The cofinality $\operatorname{cof} \alpha$ of an ordinal $\alpha$ is $\min \{|A|: A$ is an unbounded subset of $\alpha\}$; we call an infinite cardinal $\lambda$ regular if $\operatorname{cof} \lambda=\lambda$, otherwise we call it singular. (Note cof $\aleph_{\alpha+1}=$ $\aleph_{\alpha+1}, \operatorname{cof} \aleph_{0}=\aleph_{0}, \operatorname{cf} \aleph_{\omega}=\aleph_{0}$, and for limit ordinal $\left.\delta, \operatorname{cof} \aleph_{\delta}=\operatorname{cf} \delta\right)$. We write $\alpha, \beta, \gamma, \delta$ for ordinals, with $\delta$ typically a limit ordinal, and $\kappa, \lambda, \mu$ for cardinals. $\aleph_{\alpha}$ is the $\alpha$ th infinite cardinal. The first cardinal above $\lambda$ is denoted $\lambda^{+}\left(\aleph_{\alpha}^{+}=\aleph_{\alpha+1}\right)$.

The product of a set of cardinals is the cardinality of their cartesian product (with each cardinal thought of as a set of ordinals). Exponentiation is treated as a special case of the infinite product. We recall that for any ordinal $\alpha$, cof $\alpha$ is a regular cardinal, and that $\aleph_{\alpha}$ is singular iff $\alpha$ is a limit ordinal with cof $\alpha<\aleph_{\alpha}$. A cardinal $\lambda$ is a limit cardinal [strong limit cardinal] if $\mu<\lambda$ implies $\mu^{+}<\lambda\left[2^{\mu}<\lambda\right]$. Throughout our discussion the single most interesting limit cardinal will be $\aleph_{\omega}$, as was already illustrated in the introduction.

As has been known from time immemorial, addition and multiplication of two cardinals trivializes when at least one of them is infinite, so the theory of cardinal arithmetic begins with cardinal exponentiation, and more generally with infinite products. In particular, the function $\lambda \mapsto 2^{\lambda}$ satisfies the following two classical laws (the first being entirely trivial):

$$
\text { If } \mu \leq \lambda \text { then } 2^{\mu} \leq 2^{\lambda} \text {. }
$$

$$
\operatorname{cof} 2^{\lambda}>\lambda
$$

The most basic problem in (conventional) cardinal arithmetic would be whether this function obeys other laws, and for this one quickly comes to consider the behavior of $2^{\aleph_{\delta}}$ for $\delta$ a limit ordinal (as the historical discussion in $\S 2$ explains). In this case one has various relations of the type

$$
2^{\aleph_{\delta}}=\prod_{\alpha<\delta} 2^{\aleph_{\alpha}}
$$


In particular, if $\aleph_{\delta}$ is a strong limit cardinal - that is, if $2^{\aleph_{\alpha}}<\aleph_{\delta}$ for all $\alpha<\delta$ - we have

$$
2^{\aleph_{\delta}}=\aleph_{\delta}^{\operatorname{cof} \delta} .
$$

One of the difficulties in the study of cardinal arithmetic is the preoccupation with $2^{\lambda}$, or more generally with exponentiation of a small base to a large exponent; the reverse situation is considerably more manageable, and a preoccupation with strong limit cardinals is merely an attempt to trade in one problem for the other via a relation of type (3b). Maybe this preoccupation is a vestige of the Generalized Continuum Hypothesis.

We return to the first shift in viewpoint discussed in the introduction. If $\aleph_{\omega}$ is a strong limit cardinal then $2^{\aleph_{\omega}}=\aleph_{\omega}{ }^{\aleph_{0}}$, by $(3 \mathrm{~b})$. In the sample Theorem A given in the introduction, we explicitly included the hypothesis that $\aleph_{\omega}$ is a strong limit cardinal. In fact, the theorem is cleaner without it:

Theorem $\mathbf{A}^{\prime}$. $\aleph_{\omega}{ }^{\aleph_{0}}<\max \left\{\aleph_{\omega_{4}},\left(2^{\aleph_{0}}\right)^{+}\right\}$.

This is clearly a more "robust" statement than the original formulation, in that fewer extraneous considerations are involved. Unfortunately the meaning of the statement will still depend on the value of $2^{\aleph_{0}}$, the dependence being trivial if $2^{\aleph_{0}}>\aleph_{\omega}$, and we will have to work harder, beginning in $\S 4$, to eliminate this feature.

The following ideas belong to modern cardinal arithmetic, though absent from the classical theory. There is a natural topology on the class of all ordinals, in which a limit ordinal $\alpha$ is a limit point of any set $X$ of ordinals for which $X \cap \alpha$ is unbounded below $\alpha$. If $\alpha$ is an ordinal of uncountable cofinality, $\mathscr{F}_{\alpha}$ will denote the filter generated by the closed unbounded subsets of $\alpha$. This filter is closed under countable intersections.

A filter on a set $I$ is a collection of subsets of $I$ closed upward (with respect to inclusion) and closed under intersection. We require filters to be nontrivial, that is, the empty set may not be in the filter.

If $\left\{X_{i}: i \in I\right\}$ is an indexed family of sets and $\mathscr{F}$ is any filter on $I$, then the reduced product $\prod_{i} X_{i} / \mathscr{F}$ is the set of functions $f \in \prod_{i} X_{i}$ modulo the equivalence relation $=\mathscr{F}$ defined by $f=\mathscr{F} g$ iff $\{i: f(i)=g(i)\} \in \mathscr{F}$. Similarly, if the $X_{i}$ are linearly ordered, then $\prod_{i} X_{i}$ is partially ordered by pointwise comparison and $\prod_{i} X_{i} / \mathscr{F}$ is partially ordered by pointwise comparison modulo $\mathscr{F}$.

A maximal (nontrivial) filter is also called an ultrafilter and the corresponding reduced products are called ultraproducts. For our purposes, the main point is that an ultraproduct of linearly ordered sets is again linearly ordered and not just partially ordered.

We will touch lightly on issues connected with large cardinals and inner models. The reader who is unfamiliar with these subjects may ignore these remarks. On the other hand the following comments may be sufficient by way of background. There are various axioms concerning the existence of "large" cardinals (bearing names like: strongly inaccessible, measurable, supercompact, huge), which are easily seen to be unprovable on the basis of ZFC; even their consistency is known to be unprovable on the basis of ZFC, though these axioms are generally thought to be consistent. Some consistency results have been obtained only on the basis of the assumed consistency of large cardinal axioms, and the 
application of "inner models theory" is a method to prove that such consistency results require large cardinals. As our main concern is with provable theorems, this takes us rather far afield, but there is a constant interaction between the search for new theorems and the limitations imposed by independence results, which one cannot ignore in practice. In any case, when we refer to large cardinals, it is understood in that sense. When we wish to refer to cardinals that are large in a less problematic sense (bigger than some particular cardinal) we will refer to them as "moderately large."

\section{HISTORY}

Until 1974, the classical monotonicity and cofinality restrictions (1), (2) given above exhausted the known properties of the function $\left(\lambda \mapsto 2^{\lambda}\right)$. Gödel proved the consistency (with ZFC) of the generalized continuum hypothesis: $2^{\aleph_{\alpha}}=$ $\aleph_{\alpha+1}$. In 1963 Cohen introduced the notion of forcing, setting off the wave of independence results that continues to this day, and used it to prove that $2^{\aleph_{0}}$ can be any cardinal of uncountable cofinality. Easton adapted this method to show that for any appropriate function $f(\lambda)$ satisfying the monotonicity and cofinality restrictions, it is consistent to assume $2^{\lambda}=f(\lambda)$ for all regular $\lambda$. In Easton's constructions, for $\lambda$ singular $2^{\lambda}$ will always be the least value consistent with (1), (2), and the values of $2^{\mu}$ for $\mu$ regular. For example, if Easton made $\aleph_{\omega}$ a strong limit cardinal, then he made $2^{\aleph_{\omega}}=\aleph_{\omega+1}$.

Thus the belief grew that cardinal arithmetic lay outside the realm of mathematical investigation, and to complete the picture it apparently remained only to modify Easton's approach to incorporate singular cardinals. Under large cardinal hypotheses, progress was made first for large singular cardinals, and then by Magidor in 1973 for $\aleph_{\omega}$, proving for example that one could have $\aleph_{\omega}$ a strong limit and $2^{\aleph_{\omega}}=\aleph_{\omega+2}$.

So it came as a great surprise in 1974 when Silver produced a new theorem of cardinal arithmetic: if $2^{\aleph_{\alpha}}=\aleph_{\alpha+1}$ for all countable $\alpha$, then $2^{\aleph_{\omega_{1}}}=\aleph_{\omega_{1}+1}$. At this point we will leave the later independence results aside (Magidor, Foreman, Woodin, Gitik, Cummings, and the present author), as well as the complementary work on inner models and consistency strength (Jensen, Devlin, Dodd, Mitchell, Gitik), and earlier works (Scott, Solovay and Magidor) and concentrate on theorems provable in ZFC. The next section concentrates on singular cardinals of uncountable cofinality; one can skip over this development and continue in $\S 4$.

\section{3. $\lambda^{N_{1}}$}

In what follows, $\aleph_{1}$ can be replaced by any uncountable regular cardinal, but not (in this section) by $\omega$.

Answering a question of Silver, Galvin and Hajnal proved

Theorem [GH]. Suppose that $\delta$ is a limit ordinal of cofinality $\aleph_{1}$, and $\aleph_{\alpha}{ }^{\aleph_{1}}<\aleph_{\delta}$ for $\alpha<\delta$. Then $\aleph_{\delta}^{\aleph_{1}}<\aleph_{\left(2^{|| \mid} \mid\right)^{+}}$.

More precisely, if we define $f: \delta \rightarrow \delta$ by $\aleph_{\alpha}{ }^{\aleph_{1}}=\aleph_{f(\alpha)}$, the theorem states that $\aleph_{\delta}{ }^{N_{1}}<\aleph_{\|f\|}$, where $\|f\|$ is defined inductively as

$$
\sup \left\{\|g\|+1: g<f \bmod \mathscr{F}_{\delta}\right\} .
$$


(For notation, cf. §1.) This definition turns out to make sense because the filter $\mathscr{F}_{\delta}$ is closed under countable intersections. Following Silver, this line was developed in parallel and subsequent works by Baumgartner, Galvin and Hajnal, Jech and Prikry, Magidor, and the author.

When I became interested in the subject, I saw a great deal of activity and suspected I had come into the game too late; shortly thereafter I seemed to be the only one still interested in getting theorems in ZFC. I believed that the following thesis would be fruitful.

Thesis. For $\lambda$ of cofinality $\aleph_{1}$ satisfying

$$
\mu^{\aleph_{1}}<\lambda \text { for all } \mu<\lambda
$$

if $\lambda$ is small in any sense then $\lambda^{\aleph_{1}}$ is small in a related sense.

In practice this means one writes $\lambda=F_{1}(\alpha)$ for $F_{1}$ some natural function, and proves a bound $\lambda^{\aleph_{1}}<F_{2}(\alpha)$ where $F_{2}(\alpha)=F_{1}\left(\left(|\alpha|^{\aleph_{1}}\right)^{+}\right)$or some quite similar function.

For example,

Theorem [Sh 111]. If $\lambda>2^{2^{\aleph_{1}}}$ satisfies $(*)$ and is below the first regular uncountable limit cardinal, then so is $\lambda^{\aleph_{1}}$. We also get results in this vain for $F_{1}(\alpha)=$ the $\alpha$ th fix point (i.e., cardinal $\lambda$ equal to $\aleph_{\lambda}$ ).

This uses a rank function similar to Galvin/Hajnal's, with respect to more (normal) filters, which we show is well defined unless Jensen's work trivializes the problem.

We got a similar bound in [Sh 256] when $\lambda$ is the first fixed point of order $\omega$ and cofinality $\aleph_{1}$ in the enumeration of the cardinals, solving a problem raised by Hajnal following [Sh 111]. There the ranks were with respect to more complicated objects than normal filters, and in [Sh 333] similar bounds are obtained for functions defined inductively. We also prove that if the problem is not trivial then if we collapse $2^{2^{\alpha_{1}}}$ there is an ultrapower of the old universe in which for all regular $\lambda>2^{2^{N_{1}}}$ there is a $\lambda$-like element in the ultrapower (in [Sh 111] this was done for each $\lambda$ seperately). In [Sh 386] $\lambda>2^{2^{\mathrm{N}_{1}}}$ was replaced in the theorem above by $\lambda>2^{\aleph_{1}}$. A posteriori the line [Sc, So, Si, GH, Sh 111, Sh 256, Sh 386] is quite straight. The rest follows a different line.

\section{Possible cofinalities}

Although Cohen and Easton showed us that powers of regular cardinals are easily manipulated, we learned from inner model theory that this is not the case for powers of singular cardinals. Similarly, manipulating $\lambda^{\kappa}$ for $\lambda>2^{\kappa}$ is much harder than manipulating $2^{\kappa}$; the same applies to products of relatively few moderately large cardinals. There were indications [Sh b, Chapter XIII, $\S \S 5,6]$ that cofinalities are at work behind the scenes. At a certain point we began to feel that we could split off the independence results from the hard core of truth by shifting the focus.

Let $\mathbf{a}=\left(\lambda_{i}\right)_{i \in I}$ be an indexed set of regular cardinals with each $\lambda_{i}$ greater than $|I|$. Where cardinal arithmetic is concerned with the cardinality of $\Pi \mathbf{a}=$ $\prod_{i} \lambda_{i}$, we will be concerned with the following cardinal invariants involving more of the structure of the product. 
4.1. Definition. 1. A cardinal $\lambda$ is a possible cofinality of $\prod$ a if there is an ultrafilter $\mathscr{F}$ on a for which the cofinality of $\Pi \mathbf{a} / \mathscr{F}$ is $\lambda$. (Recall that these ultraproducts are linearly ordered: $\S 1$.)

2. pcf $\mathbf{a}$ is the set of all possible cofinalities of $\Pi \mathbf{a}$.

We first used pcf in [Sh 68] in a more structural context, to construct Jońsson algebras (algebras of a given cardinality with no proper subalgebra of the same cardinality). In [Sh b, Chapter XIII, $\S \S 5,6]$ we obtained results under the more restrictive hypothesis $\lambda_{i}>2^{|\mathbf{a}|}$ bearing on cardinal arithmetic. An instance of the main theorem there is the following (note that $[\mathrm{GH}]$ did not give information on cardinals with countable cofinalities, Theorem $\mathrm{A}$ is a significant improvement though formally they are incomparable).

\subsection{Theorem $\mathbf{B}^{\prime}$. If $2^{\aleph_{0}}<\aleph_{\omega}$ then $\aleph_{\omega}{ }^{\aleph_{0}}<\aleph_{\left(2^{\left.\aleph_{0}\right)^{+}}\right.}$.}

Since there are $2^{2^{|I|}}$ ultrafilters on $I$, pcf a could be quite large a priori; this would restrict heavily its applications to cardinal arithmetic. Fortunately there are various uniformities present that lead to a useful structure theory for pcf.

4.3. Main Theorem. Let $\mathbf{a}=\left(\lambda_{i}\right)_{i \in I}$ be an indexed set of distinct regular cardinals, with each $\lambda \in \mathbf{a}$ greater than $|\mathbf{a}|$. Then:

1. pcf a contains at most $2^{|\mathbf{a}|}$ cardinals;

2. pcf a has a largest element max pcf a ;

3. $\operatorname{cof} \prod \mathbf{a}=\max$ pcf $\mathbf{a}$ (see remark below);

4. For each $\lambda \in \mathbf{p c f} \mathbf{a}$ there is a subset $\mathbf{b}_{\lambda}$ of $\mathbf{a}$ such that

a. $\lambda=\max \operatorname{pcf} \mathbf{b}_{\lambda}$, and

b. $\lambda \notin \operatorname{pcf}\left(\mathbf{a}-\mathbf{b}_{\lambda}\right)$;

5. If $\mathscr{J}_{\lambda}$ is the ideal on I generated by the sets $\mathbf{b}_{\mu}$ for $\mu<\lambda$, then for each $\lambda \in \mathrm{pcf}$ a there are functions $f_{i}^{\lambda}(i<\lambda)$ such that

a. for $i<j$ we have $f_{i}^{\lambda}<f_{j}^{\lambda} \bmod \mathcal{J}^{\lambda}$;

b. for any $f \in \prod \mathbf{a}$ and $\lambda \in \operatorname{pcf}$ a there is some $i<\lambda$ such that $f<f_{i}^{\lambda} \bmod \left\langle\mathscr{J}_{\lambda},\left(\mathbf{a}-\mathbf{b}_{\lambda}\right)\right\rangle$.

Remarks. The meaning of clause (3) is that for $\lambda=\max$ pcf $\mathbf{a}$, there is a subset $P$ of $\prod$ a of cardinality $\lambda$ that is cofinal in the sense that every function in $\Pi \mathbf{a}$ is dominated pointwise by a function in $P$. For example, the cofinality of $\omega \times \omega_{1}$ is $\omega_{1}$. This does not mean that there is a pointwise nondecreasing sequence of length $\omega_{1}$ that dominates every element of $\omega \times \omega_{1}$; there is no such sequence. When a product actually contains a cofinal pointwise nondecreasing sequence, we say its cofinality is true, and we write tcf for the cofinality when it is true.

By clause (5) $\prod \mathbf{b}_{\lambda} / \mathscr{F}_{\lambda}$ has true cofinality $\lambda$.

The following structural principle is also of great practical importance. Note that we do not know whether $|\operatorname{pcf} \mathbf{a}| \leq|\mathbf{a}|$, the following still says that pcf $\mathbf{a}$ is "small," "local," has small density character.

4.4. Localization Theorem. Let a be a set of $\kappa$ distinct regular cardinals with $\lambda>\kappa$ for all $\lambda \in \mathbf{a}$; and suppose $\mathbf{b} \subseteq$ pcf a with $\lambda>|\mathbf{b}|$ for all $\lambda$ in $\mathbf{b}$. If $\mu \in \operatorname{pcf} \mathbf{b}$ then $\mu \in \operatorname{pcf} \mathbf{a}$, and for some $\mathbf{c} \subseteq \mathbf{b}$ of cardinality at most $\kappa$ we have $\lambda \in \operatorname{pcf} \mathbf{c}$. 
Thus pcf defines a canonical closure operation on sets of regular cardinals with some good properties.

\section{PSEUdOPOWERS}

For $\operatorname{cof} \lambda \leq \kappa<\lambda$ we define the pseudopower $\mathrm{pp}_{\kappa}(\lambda)$ as follows.

5.1. Definition. 1. $\mathrm{pp}_{\kappa}(\lambda)$ is the supremum of the cofinalities of the ultraproducts $\prod \mathbf{a} / \mathscr{F}$ associated with a set of at most $\kappa$ regular cardinals below $\lambda$ and an ultrafilter $\mathscr{F}$ on a containing no bounded set bounded below $\lambda$.

2. $\mathrm{pp}(\lambda)$ is $\mathrm{pp}_{\operatorname{cof} \lambda}(\lambda)$.

If we have a model of set theory with a very interesting (read: bizarre) cardinal arithmetic, say $2^{\aleph_{\omega}}=\aleph_{\omega+2}$, and we adjust $2^{\aleph_{0}}$ by Cohen's method, putting in $\aleph_{\omega+3}$ Cohen reals, there is then no nontrivial operation of cardinal arithmetic that will yield the result $\aleph_{\omega+2}$. Not that the original phenomena have been erased; they are simply drowned out by static. The operation $\mathrm{pp}_{\kappa}(\lambda)$ is more robust. It will be convenient to call $\lambda \kappa$-inaccessible if $\mu^{\kappa}<\lambda$ for $\mu<\lambda$.

5.2. Theorem. 1. $\mathrm{pp}_{\kappa}(\lambda) \leq \lambda^{\kappa}$;

2. If $\operatorname{cof} \lambda \leq \kappa<\lambda<\aleph_{\lambda}$ and $\lambda$ is $\kappa$-inaccessible (i.e., $\left.(\forall \mu<\lambda)\left[\mu^{\kappa}<\lambda\right]\right)$, then $\operatorname{pp}_{\kappa}(\lambda)=\lambda^{\kappa}$.

In this sense, $\operatorname{pp}_{\kappa}(\lambda)$ is an antistatic device. If $\lambda$ is $\kappa$-inaccessible in one model of set theory and this condition is then destroyed in an extension in a reasonably subdued manner (i.e., when we make $2^{\kappa}$ large), $\mathrm{pp}_{\kappa}(\lambda)$ continues to reflect the earlier value of $\lambda^{\kappa}$. On another level, one can prove results about this operation by induction on cardinals, which is not possible with the less robust notions. (The restriction $\lambda<\aleph_{\lambda}$ is certainly convenient, but we will discuss its removal below.)

Let $\operatorname{PP}_{\kappa}(\lambda)$ be the set of cofinalities whose supremum was taken to get $\mathrm{pp}_{\kappa}(\lambda)$. This turns out to have the simplest possible structure.

5.3. Convexity Theorem. If $\kappa \in[\operatorname{cof} \lambda, \lambda)$ then $\operatorname{PP}_{\kappa}(\lambda)$ is an interval in the set of regular cardinals with minimum element $\lambda^{+}$.

We can also give a cleaner description of the way a cardinal enters $\operatorname{PP}_{\kappa}(\lambda)$ in some cases.

5.4. First Representation Theorem. Suppose $\aleph_{0}<\operatorname{cof} \lambda<\kappa<\lambda$ and for all $\mu \in(\kappa, \lambda)$; if $\operatorname{cof} \mu \leq \operatorname{cof} \lambda$ then $\operatorname{pp}(\mu)<\lambda$. Let $\mathscr{F}_{0}$ be the filter on $\operatorname{cof} \lambda$ generated by the complements of the bounded sets. Then for any regular cardinal $\lambda^{*} \in \operatorname{PP}(\lambda)$, there is an increasing sequence $\left(\lambda_{i}\right)_{i<\operatorname{cof} \lambda}$ with limit $\lambda$ such that the product $\prod \lambda_{i} / \mathscr{F}_{0}$ has true cofinality $\lambda^{*}$.

In the case $\lambda^{*}=\lambda^{+}$there is a better representation.

5.5. Second Representation Theorem. If $\lambda$ is singular of uncountable cofinality, $\mathscr{F}_{0}$ is the filter generated by the complements of the bounded subsets of cof $\lambda$, and $\left(\lambda_{i}\right)_{i<\operatorname{cof} \lambda}$ is increasing, continuous and cofinal in $\lambda$, then there is a closed unbounded subset $C$ of $\operatorname{cof} \lambda$ such that $\prod_{C} \lambda_{i}^{+} /\left\langle\mathscr{F}_{0}\right.$, cof $\left.\lambda-C\right\rangle$ has true cofinality $\lambda^{+}$.

Discussion. Why do we suggest $P P_{\kappa}(\lambda)$ as a replacement to $\lambda^{\kappa}$ ? Maybe we had better reconsider what we are doing. $\lambda^{\kappa}$ is a measure of the size of the family of subset $\lambda$ of cardinality $\leq \kappa$, i.e., its cardinality. Remember that when 
$\lambda \leq \kappa$ the independence results do not leave us much to say. When $\kappa<\lambda$ we shall present various natural measures of this set below. It is useful to prove that various such measures are equal, as we can then look at them as various characterizations of the same number each useful in suitable circumstances. We report reasonable success in this direction below.

5.6. Definition. For $\kappa \in[\operatorname{cof} \lambda, \lambda)$ let the $\kappa$-covering number for $\lambda, \operatorname{cov}(\lambda, \kappa)$, be the minimal cardinality of a family of subsets of $\lambda$, each of size less than $\lambda$, such that every subset of $\lambda$ of size $\kappa$ is contained in one of the specified sets. Note that, as is well known, for $\kappa<\lambda, \lambda^{\kappa}=2^{\kappa}+$ "the $\kappa$-covering of $\lambda$."

5.7. Theorem. If $\kappa \in[\operatorname{cof} \lambda, \lambda)$ and $\lambda<\aleph_{\lambda}$ then $\mathrm{pp}_{\kappa}(\lambda)$ is the $\kappa$-covering number for $\lambda$.

To remove the restriction that $\lambda<\aleph_{\lambda}$, define the weak $\kappa$-covering number for $\lambda$ as the minimal cardinality of a family of subsets of $\lambda$, each of size less than $\lambda$, such that every subset of $\lambda$ of size $\kappa$ is contained in the union of countably many of the specified sets.

5.8. Theorem. 1. For $\kappa$ uncountable, the weak $\kappa$-covering number of $\lambda$ is the supremum of the true cofinalities of the reduced products $\prod \mathbf{a} / \mathscr{F}$ with a a set of at most $\kappa$ regular cardinals below $\lambda$ and $\mathscr{F}$ a filter on a that contains the complement of every subset of a bounded below $\lambda$ and is closed under countable intersections.

2. If this cardinal is regular, the indicated supremum is attained.

Thus our problems are connected with the case of cofinality $\omega$. We give relevant partial information in $\S 8$.

We mention two more invariants that we can now prove coincide.

5.9. Theorem. For $\aleph_{0}<\kappa \leq \lambda$ with $\kappa$ regular the following two cardinals coincide:

1. The minimal cardinality of a family of subsets of $\lambda$, each of size less than $\kappa$, such that any subset of $\lambda$ of cardinality less than $\kappa$ is contained in one of the specified sets.

2. The minimal cardinality of a stationary subset of the family of subsets of $\lambda$ of size less than $\kappa$. (Stationarity means that for every algebra with set of elements $\lambda$ and countably many operations, there is a subalgebra $B,|B|<\kappa$, $B \cap \kappa$ is a ordinal and $B \in S$.)

\section{CARDINAL ARITHMETIC REVISITED}

If $\kappa=\aleph_{\alpha}$, it will be convenient to call $\alpha$ the index of $\kappa$; otherwise our results tend to live entirely in the land of subscripts. In [Sh b, Chapter XIII, $\S \S 5,6]$ we showed

$$
\text { The index of } \aleph_{\delta}^{\operatorname{cof} \delta} \text { is less than }\left(|\delta|^{\operatorname{cof} \delta}\right)^{+} .
$$

In particular, for $\delta=\omega$, the corresponding index is below $\left(2^{\aleph_{0}}\right)^{+}$(construed as an ordinal). If $\aleph_{\omega}$ is itself below $2^{\aleph_{0}}$ then this contains no information and (our usual theme) inessential modifications of the universe can always make this happen. On the other hand known independence results show that when $\aleph_{\omega}$ is a strong limit, the index of $\left(\aleph_{\omega}\right)^{\aleph_{0}}$ can be any countable successor ordinal, so in principle one could hope to prove that the true bound is $\omega_{1}$ (which we doubt). Our strongest result is 
6.1. Theorem. $p p\left(\aleph_{\omega}\right)<\aleph_{\omega_{4}}$.

6.2. Corollary. $\aleph_{\omega}^{\aleph_{0}}<\max \left(\aleph_{\omega_{4}},\left(2^{\aleph_{0}}\right)^{+}\right)$.

The more general formulation is

6.3. Theorem. If $\delta$ is a limit ordinal, $\delta<\aleph_{\alpha+\delta}$ then $\operatorname{pp}\left(\aleph_{\alpha+\delta}\right)<\aleph_{\alpha+|\delta|^{+4}}$.

For the proof, one looks carefully at the closure operation induced on $\omega_{4}$ by the pcf structure on $\aleph_{\omega_{4}}$ (passing to indices) under the assumption that $\aleph_{\omega_{4}}$ is $\leq p p\left(\aleph_{\omega}\right)$. There are conflicts between the main theorem, the localization theorem and the second representation theorem on the one hand and combinatorics of closed unbounded sets inside $\omega_{4}$ on the other hand, which yield a contradiction. Ultimately, the pcf structure cannot exist on $\omega_{4}$ because no such closure operation exists. On $\omega_{3}$, there are two questions: does such a closure operation exist and can it be given by pcf?

Structure of the proof. Assuming (toward a contradiction) that $\aleph_{\omega_{4}+1}$ belongs to $\operatorname{pcf}\left(\aleph_{\omega}\right)$, for $X \subseteq \omega_{4}$ define $\operatorname{cl} X=\left\{i<\omega_{4}: \aleph_{i+1} \in \operatorname{pcf}\left(\aleph_{j+1}\right)_{j \in X}\right\}$. By the Convexity Theorem $\operatorname{cl}(\omega)=\omega_{4}$ and by the Localization Theorem, if $i \in \operatorname{cl} X$ then for some countable $Y \subseteq X$ we have $i \in \operatorname{cl} Y$. By the Second Representation Theorem, if $\delta<\omega_{4}$ is a limit ordinal of uncountable cofinality then $\delta$ is the maximal element of $\operatorname{cl} C$ for some closed unbounded subset of $\delta$ as well as any smaller closed unbounded set. These three properties of the closure operation (alone) eventually lead to a contradiction.

This may indicate that it is interesting to investigate, e.g., the set $\left\{\aleph_{\alpha+1}\right.$ : $\left.\aleph_{\alpha+1} \leq \operatorname{pp} \aleph_{\omega}\right\}=\operatorname{pcf}\left\{\aleph_{n}: n\right\}$ with the relation " $\lambda \in \operatorname{pcfb}$ " and even the sequence $\left\langle\mathbf{b}_{\lambda}: \lambda \in \operatorname{pcf}\left\{\aleph_{n}: n\right\}\right\rangle$ from 4.3 (for which there are theorems saying "we can choose nicely").

\section{FOR TRUE BELIEVERS}

Naturally our results also give a great deal of information regarding the types of forcing that are applicable to certain open questions. Any instance of $\lambda \in$ pcf a normally results from some normal ultrafilter "at the time when the large cardinals were present." Some of this information is in the canonical spot in [Sh-b, Chapter XIII, $\S \S 5,6]$, and more is in [Sh 282], for example, if $\operatorname{pp}\left(\aleph_{\omega_{1}}\right)$ is greater than $\aleph_{\omega_{2}}$ then there are many ordinals $\delta<\omega_{2}$ of cofinality $\omega$ for which $\operatorname{pp}\left(\aleph_{\delta}\right)$ is above $\aleph_{\delta+\omega_{1}}$.

These considerations also shed some light on the problem of resurrection of supercompactness. By our 9.6(1)

7.1. Corollary. If in $V$ we have $\operatorname{cof}(\lambda)<\kappa<\lambda$ and $\operatorname{pp}(\lambda)>\lambda^{+}$, then there is no universe extending $V$ in which $\lambda^{+}$remains a cardinal while $\kappa$ becomes supercompact or even compact.

Gitik has proved, for example, that if $\operatorname{cof} \lambda=\omega, \mu^{\aleph_{0}}<\lambda$ for all $\mu<\lambda$, and $\operatorname{pp}(\lambda)>\lambda^{+}$, then in Mitchell's inner model $o(\lambda)=\lambda^{++}$. By his previous independence results this settles the consistency strength of $2^{\aleph_{0}}<\aleph_{\omega+1}<\aleph_{\omega} \aleph_{0}$ (using [Sh b]), though it did not settle the consistency strength of the full singular cardinal hypothesis, as there are fixed points. For this purpose we have proved

7.2. Lemma. If $\lambda$ is singular of cofinality $\aleph_{0}$, and if $\mathrm{pp}(\mu)<\lambda$ for all singular cardinals $\mu<\lambda$ of cofinality $\aleph_{0}$ and $p p(\mu)=\mu^{+}$for every large enough $\mu<\lambda$ 
of cofinality $\aleph_{1}$, then there is a family of countable subsets of $\lambda$ containing $p p(\lambda)$ sets, so that every countable subset of $\lambda$ is contained in one of the specified sets. Thus if $\mu^{\aleph_{0}}<\lambda$ for all $\mu<\lambda$, we find $\mathrm{pp}(\lambda)=\lambda^{\aleph_{0}}$.

Together this yields an equiconsistency result. However Gitik points out that this result is intrinsically global. For a localized result, we have

7.3. Lemma. If $\lambda$ is singular of cofinality $\aleph_{0}$, and if $\mu^{\aleph_{0}}<\lambda$ for $\mu<\lambda$ and $\operatorname{pp}(\lambda)>\lambda^{\aleph_{0}}$, then $\mathrm{pp}(\lambda)$ is quite large; there are at least $\aleph_{1}$ fixed points in the interval from $\lambda^{+}$to $\mathrm{pp}(\lambda)$.

This still does not settle the behavior of singular cardinals of cofinality $\aleph_{0}$. The following suggests there are few exceptional values.

7.4. Lemma. If $\kappa$ is a regular uncountable cardinal and $\left(\lambda_{i}\right)_{i \leq \kappa}$ is an increasing continuous sequence of cardinals with $\lambda_{i}^{\kappa}<\lambda_{\kappa}$ for $i<\kappa$, then for some closed unbounded subset $C$ of $\kappa$ we have $\mathrm{pp}\left(\lambda_{i}\right)=\lambda_{i}^{\kappa}$ on $C \cup\{\kappa\}$.

This has application to the construction of "black boxes."

\section{SOME HYPOTHESES}

\subsection{The Strong Hypothesis. For all singular cardinals $\lambda, p p(\lambda)=\lambda^{+}$.}

This is a replacement for $\mathrm{GCH}$ as well as " $0^{\#}$ does not exist" in some cases. It is weaker than either and is hard to change by forcing, but is consistent with large cardinals (and indeed holds above any compact cardinal [So] while having a more combinatorial character than " $\neg 0^{\#}$ ". Of course this will not give, e.g., a square sequence on the successor of a singular cardinal.)

8.2. Lemma. The strong hypothesis implies that for any singular cardinal $\lambda$ and any $\kappa<\lambda, \lambda^{\kappa} \leq \lambda^{+}+2^{\kappa}$, there are $\lambda^{+}$subsets of $\lambda$, each of cardinality $\kappa$, such that every subset of $\lambda$ of cardinality $\kappa$ is contained in one of the specified sets; and a Jonsson algebra exists in every successor cardinal.

8.3. The Weak Hypothesis. For any singular cardinal $\lambda$, there are at most countably many singular cardinals $\mu<\lambda$ with $\operatorname{pp}(\mu) \geq \lambda$.

In my opinion, it is a major problem to determine whether this follows from $\mathrm{ZFC}$ and is the real problem behind the determination of the true bound on $\mathrm{pp}\left(\aleph_{\omega}\right)$.

8.4. Lemma. The weak hypothesis implies:

- $\operatorname{pp}\left(\aleph_{\omega}\right)<\aleph_{\omega_{1}}$, and more generally if $\delta<\aleph_{\delta}$ then $\mathrm{pp}\left(\aleph_{\delta}\right)<\aleph_{|\delta|^{+}}$.

- $\mathbf{p c f}$ a has cardinality at most $|\mathbf{a}|$.

- $\operatorname{pp}(\lambda)$ has cofinality at least $\lambda^{+}$for $\lambda$ singular.

If we strengthen the weak hypothesis by replacing "countable" by finite, but only for $\mu$ of cofinality $\aleph_{0}$, then Gitik has proved that the stronger version does not follow from ZFC.

\section{OTHER APPLiCATIONS}

If the list of applications below does not contain "familiar faces" the reader will not lose by skipping to the concluding remarks. 
We turn now to results involving more structural information, bearing on almost free abelian groups, partition problems, failure of preservation of chain conditions in Boolean algebras under products, existence of Jońsson algebras, existence of entangled linear orders, equivalently narrow Boolean algebras, and the existence of $L_{\infty, \lambda}$-equivalent nonisomorphic models.

9.1. Model theory. If $\lambda$ has cofinality greater than $\aleph_{1}$ then there are two $L_{\infty, \lambda^{-}}$ equivalent nonisomorphic models of cardinality $\lambda$.

This was known for $\lambda$ regular or $\lambda=\lambda^{\aleph_{0}}$, and for strong limit $\lambda$ of uncountable cofinality, but fails for cofinality $\aleph_{0}$. There is still a small gap.

\subsection{Jońsson Algebras. There is a Jońsson algebra of cardinality $\aleph_{\omega+1}$.}

A Jońsson algebra is an algebra in a finite or countable language that has no proper subalgebra of the same cardinality.

This was known previously under the hypothesis $2^{\aleph_{0}} \leq \aleph_{\omega+1}$. We can now show that if $\lambda$ is singular and there is no Jońsson algebra of cardinality $\lambda^{+}$, then $\lambda$ is quite large, for example, $\lambda$ is a limit of weakly inaccessible cardinals that do not admit Jońsson algebras (and there is a Jońsson algebra of inaccessible cardinality $\mu$ if $\mu$ is not Mahlo, or even not $(\mu \times \omega)$-Mahlo).

9.3. Chain conditions. For any cardinal $\lambda>\aleph_{1}$, there is a boolean algebra that satisfies the $\lambda^{+}$-chain condition while its square does not; hence its Stone-Čch compactification has cellularity $\lambda$, but its square has cellularity greater than $\lambda$

Previous References: [T1, Sh 282] for some singular cases, [Sh 280] for regular above $2^{\aleph_{0}}$, [Sh 327] for regular $\lambda \geq \aleph_{2}$.

This really comes from coloring theorems. We give a sample of the latter.

9.4. Coloring Theorem. For $\lambda>\aleph_{1}$ there is a binary symmetric function $c$ : $\left(\lambda^{+}\right)^{2} \rightarrow \operatorname{cof} \lambda$ such that for any sequence $\left(w_{i}\right)_{i<\lambda}$ of pairwise disjoint finite subsets of $\lambda^{+}$and any $\gamma<\operatorname{cof} \lambda$, there is a pair $i<j<\lambda$ with $c\left[w_{i} \times w_{j}\right]=\{\gamma\}$.

9.5. Narrow Boolean algebras. 1. If $\lambda$ is singular and less than $2^{\aleph_{0}}$, then there is a $\lambda^{+}$-narrow Boolean order algebra of cardinality $\lambda^{+}$(see below). The same holds if $\kappa^{+3}<\operatorname{cof} \lambda<\lambda<2^{\kappa}$.

2. The class $\left\{\lambda\right.$ : there is in $\lambda^{+}$a $\lambda^{+}$-narrow Boolean algebra $\}$is not bounded (really $\left(\lambda, \aleph_{\lambda+3+1}\right]$ is not disjoint to it).

9.5A. Definitions. 1. The boolean order algebra associated to a linear order $L$ is the boolean algebra of subsets of $L$ generated by the closed-open intervals.

2. A boolean algebra is $\lambda$-narrow if it has no set of pairwise incomparable elements of size $\lambda(a, b$ are incomparable if $a \leq b b$ and $b \leq z a)$.

3. A linear order $L$ is entangled if for every $n$, for all choices of distinct $x_{m, i}$ for $m \leq n, i<|L|$, and for any subset $w$ of $\{1, \ldots, n\}$, there are $i<j$ so that for all $m \leq n$ we have $x_{m, i}<x_{m, j}$ iff $m \in w$.

For $\lambda$ regular, the boolean order algebra associated to the linear order $L$ of cardinality $\lambda$ is $\lambda$-narrow if and only if $I$ is entangled. For background see [ARSh 153, BoSh 210, T, Sh 345].

The bound $\lambda^{+3}$ in the theorem above is connected to $\S 6$. Note that in addition we can use a subdivision into various cases, bearing in mind that by [BSh $212, \mathrm{~T}]$ there is such an algebra of cardinality $\operatorname{cof} 2^{\lambda}$ if there is a linear order of 
cardinality $2^{\lambda}$ and density $\lambda$, and usually the absence of such an order enables us to use our current method.

9.6. Almost disjoint sets, almost free Abelian groups. 1. If $\lambda$ is singular and $\operatorname{pp}(\lambda)>\lambda^{+}$, then there is a family of $\lambda^{+}$subsets of $\lambda$, each of cardinality $\operatorname{cof} \lambda$, such that any $\lambda$ of them admit an injective choice function (called transversal). Consequently if cof $\lambda=\aleph_{0}$ then there is a $\lambda^{+}$-free uut not free abelian group.

2. The following are equivalent for $\lambda>\mu \geq 2^{\aleph_{0}}$.

a. There are $\lambda$ subsets of $\mu$ of size $\aleph_{1}$ such that any two have finite intersection.

b. For some $n$, there are regular cardinals $\lambda_{i, m}$ for $i<\omega_{1}$ and $m \leq n$, such that for every infinite subset $X$ of $\omega_{1}, \mu \leq \max \operatorname{pcf}\left(\lambda_{i, m}: i \in X, m \leq n\right)$.

3. If $\lambda$ is regular, $2^{<\lambda}<2^{\lambda}$, and there is no linear order of cardinality $2^{\lambda}$ and density $\lambda$, then in every regular $\mu \in\left[2^{<\lambda}, 2^{\lambda}\right]$ there is an entangled order.

There are other results on almost disjoint sets and $\lambda$-free abelian groups and a topological question of Gretlis, Hajnal, and Szentmiclossy.

Concluding Remarks. The following is not surprising in view of Theorem 6.3, and part of the argument is similar but requires, at least from the author, considerably more work.

Theorem. If $\delta<\aleph_{\omega_{4}}$ has cofinality $\aleph_{0}$, then $\mathrm{pp}\left(\aleph_{\delta}\right)<\aleph_{\omega_{4}}$, and hence the cofinality of the partially ordered set $\left\langle S_{\leq \aleph_{0}}\left(\aleph_{\omega_{4}}\right) ; \subseteq\right\rangle$ is $\aleph_{\omega_{4}}$ (where $S_{\aleph_{0}}(\lambda)=$ $\left.\left\{a: a \subseteq \lambda,|a|=\aleph_{0}\right\}\right)$.

Also the state of Tarski conjecture can be clarified, by Jech and Shelah [JeSh 385] and, e.g., if $p p\left(\aleph_{\omega_{1}}\right)>\aleph_{\omega_{2}}$ then there is a Kurepa tree on $\omega_{1}$.

We conclude with some words of the author. Reflecting on the above it seems that whereas once we knew a considerable amount about the uncountable cofinality case and nothing about the countable cofinality case (I mean theorems and not consistency results or consistency strength results), now the situation has reversed. This is not accurate-the results of Galvin and Hajnal and those discussed in $\S 3$ above, are not superceded by the later results. There does not seem to be a generalization of ranks to countable cofinality, nor have we suggested so far anything on fixed points or limits of inaccessibles below the contiuum. Recently we have succeed in getting some results on this topic, and they will be reported elsewhere [Sh 420]. See more in Gitik Shelah [GiSh 412] (mainly if $\lambda$ is real valued measurable $\left\{2^{\sigma}: \sigma<\lambda\right\}$ is finite), [Sh 413] (more on coloring and Jonsson algebras), [Sh 430] (on $\operatorname{cf}\left(\mathscr{S}_{<\aleph_{1}}(\lambda), \subseteq\right) \leq \lambda$ for $\lambda$ real valued measurable, and again of existence of trees with $\kappa$ nodes and any regular $\lambda \in\left[\kappa, 2^{\kappa}\right] \kappa$-branches and improvements of 7.3 and on the smallest values needed for canonization theorems).

\section{REFERENCES}

[ARSh 153] U. Abraham, M. Rubin, and S. Shelah, On the consistency of some partition theorems for continuous colorings and the structure of $\aleph_{1}$-dense real order types, Ann. Pure Appl. Logic 29 (1985), 123-206.

[B] J. Baumgartner, Almost disjoint sets, the dense set problem, and the partition calculus, Ann. Pure Appl. Logic 9 (1975), 401-439.

[BP] J. Baumgartner and K. Prikry, On a theorem of Silver, Discrete Math. 14 (1976), 17-22. 
[BoSh 210] R. Bonnet and S. Shelah, Narrow boolean algebras, Ann. Pure Appl. Logic 28 (1985), 1-12.

[C] P. Cohen, Set theory and the continuum hypothesis, Benjamin, NY, 1966. CA.

[Cu] J. Cummings, Consistency results in cardinal arithmetic, $\mathrm{Ph}$. D. thesis, Cal-Tech, Pasadena,

[CW] J. Cummings and H. Woodin, preprint.

[DeJ] K. Devlin and R. B. Jensen, Marginalia to a theorem of Silver, Proceedings of the Logic Colloquium, Kiel 1974 (G. H. Müller, A. Oberschelp, and K. Potthoff, eds.), Lecture Notes in Math., vol. 499, Springer-Verlag, Berlin, 1975, pp. 115-142.

[DoJ] A. Dodd and R. B. Jensen, The core model, Annals Math. Logic (Ann. Pure Appl. Logic) 20 (1981), 43-75. $1-35$.

[FW] M. Foreman and H. Woodin, G.C.H can fail everywhere, Ann. of Math. (2) 133 (1991),

[GH] F. Galvin and A. Hajnal, Inequalities for cardinal powers, Ann. of Math. (2) 101 (1975), 491-498.

[GHS] J. Gertlis, A. Hajnal, and Z. Szentmiklossy, On the cardinality of certain Hausdorff spaces, Frolic Memorial Volume, accepted.

[Gi] M. Gitik, The negation of SCH from $o(\kappa)^{++}$, Ann. Pure Appl. Logic 43 (1989), 209-234.

[Gil] _ The strength of the failure of the singular cardinal hypothesis, preprint.

[GM] M. Gitik and M. Magidor, The singular cardinal hypothesis revisited (in preparation).

[GiSh 344] M. Gitik and S. Shelah, On certain indestructibility of stray cardinals and a question of Hajnal, Arch. Math Logic 28 (1989), 35-42.

[GiSh 412] M. Gitik and S. Shelah, More on ideals with simple forcing notions, Ann. Pure Appl. Logic (to appear).

[JP] T. Jech and K. Prikry, Ideals over uncountable sets: applications of almost disjoint sets and generic ultrapowers, Mem. Amer. Math. Soc. 214 (1979).

[JeSh 385] T. Jech and S. Shelah, On a conjecture of Tarski on products of cardinals, Proc. Amer. Math. Soc. 112 (1991), 1117-1124.

[Mg] M. Magidor, On the singular cardinals problem I, Israel J. Math. 28 (1977), 1-31.

[Mg1] _ On the singular cardinals problem II, Ann. of Math. (2) 106 (1977), 517-547.

[Mg2] _ _ Chang's conjecture and powers of singular cardinals, J. Symbolic Logic 42 (1977), $272-276$.

[M] W. Mitchell, The core model for sequences of measures I, Math. Proc. Cambridge Philos. Soc. 95 (1984), 229-260. $1-52$.

[Pr] K. Prikry, Changing measurable to accessible cardinals, Rozprawy Mat. LXVIII (1970),

[Sh 68] S. Shelah, Jońsson algebras in successor cardinals, Israel J. Math. 39 (1978), 475-480.

[Sh 71] _ _ A note on cardinal exponentiation, J. Symbolic Logic 45 (1980), 56-66. 299.

[Sh 111] _ On powers of singular cardinals, Notre Dame J. Formal Logic 27 (1986), 263-

[Sh b] _ _ Proper Forcing, Lecture Notes in Math., vol. 940, Springer-Verlag, Berlin, 1982.

[Sh 137] _ The singular cardinal problem: independence results, Proceedings of a Symposium on Set Theory, Cambridge 1978 (A. Mathias, ed.), London Math Soc. Lecture Notes Ser., vol. 87, Cambridge Univ. Press, Cambridge and New York, 1983, pp. 116-134.

[Sh 161] _ Incompleteness in regular cardinals, Notre Dame J. Formal Logic 26 (1985), 195-228.

[Sh 256] _ _ More on powers of singular cardinals, Israel J. Math. 59 (1987), 263-299.

[Sh 280] _ Strong negative partition relations above the continuum, J. Symbolic Logic 55 (1990), 21-31.

[Sh 282] _ _ Successors of singulars, productivity of chain conditions, and cofinalities of reduced products of cardinals, Israel J. Math. 60 (1987), 146-166. 
[Sh 327] __ Strong negative partition relations below the continuum, Acta Math. Hungar. in press.

[Sh 410] _ More on cardinal arithmetic, Arch. Math. Logic (to appear).

[Sh 413] _ _ More Jonsson algebras and coloring, preprint.

[Sh 420] _ Advances in cardinal arithmetic, to appear.

[Sh 430] - Further cardinal arithmetic, preprint.

[Sh-g] _ Cardinal arithmetic, OUP, (to appear).

[ShW 159] S. Shelah and H. Woodin, Forcing the failure of the CH, J. Symbolic Logic 49 (1984), 1185-1189.

[Sc] D. Scott, Measurable cardinals and constructible sets, Bull. Acad. Pol. Sci. Ser. Math. Astron. Phys. 9 (1961), 521-524.

[Si] J. Silver, On the singular cardinal problem, Proceedings ICM, Vancouver 1974, vol. I, pp. 265-268,

[So] R. Solovay, Strongly compact cardinals and the GCH, Proceedings of the Tarski Symposium, Berkeley 1971, Proc. Sympos. Pure Math., vol. xxi, Amer. Math. Soc., Providence, RI, 1974, pp. 365-372.

[T] S. Todorčevic̀, Remarks on chain conditions in products, Compositio Math. 56 (1985), 295-302.

[T1] __ Remarks on cellularity in products, Compositio Math. 57 (1986), 357-372.

[W] H. Woodin, The Collected Unwritten Works of H. Woodin.

Mathematics Department, The Hebrew University, Jerusalem, Israel

Current address: Mathematics Department, Hill Center, Busch Campus, Rutgers-The State University, New Brunswick, New Jersey 08903 